\title{
Review of: "Eplerenone Reverses Age-Dependent Cardiac Fibrosis Through Downregulating Osteopontin"
}

Anastasios Lymperopoulos ${ }^{1}$

1 Nova Southeastern University

Potential competing interests: The author(s) declared that no potential competing interests exist.

This is an interesting study on the effects of eplerenone on cardiac fibrosis via osteopontin downregulation.

Major comments:

1. Do the authors have any data on osteopontin protein levels?

2. Is the effect of eplerenone reversed by aldosterone administration?

3. Do the authors have any data on possible involvement of the beta2 adrenergic receptor in the effects of eplerenone? The mineralocorticoid receptor, which is the target of eplerenone, is known to upregulate osteopontin and to be blocked by this receptor via GRK5 activation (PMID: 32326036). Osteopontin, in turn, blocks beta2 adrenergic receptor`s anti-fibrotic signaling in the heart (PMID: 30897705 ).

Minor comment: Shouldn 't eplerenone be abbreviated "EPL" instead of "APL" in the figures? 